\title{
MANNOSYLATED MULTIWALLED CARBON NANOTUBES ASSISTED ARTESUNATE DELIVERY FOR CEREBRAL MALARIA
}

\author{
ANAMIKA SAHU GULBAKE ${ }^{1}$, AVIRAL JAIN ${ }^{2}$, SATISH SHILPI ${ }^{3}$, PRAMOD KUMAR ${ }^{4}$, ARVIND GULBAKE ${ }^{5}$
}

1Pharmaceutics Research Laboratory, Department of Pharmaceutics, Adina Institute of Pharmaceutical Sciences, Sagar (M. P.) India, ${ }^{2}$ JKITE, Department of Pharmacy, Lucknow, U. P. India, ${ }^{3}$ Pharma Research Project Laboratory, Department of Pharmaceutics, Ravishankar College of Pharmacy, Bhopal, ${ }^{4}$ Helmholtz Zentrum München, Ingolstädter Landstraße $1 \cdot$ D-85764 Neuherberg, Germany, ${ }^{5}$ Centre for Interdisciplinary Research, D. Y. Patil Education Society, Deemed to be University, Kolhapur, M. S., 416006, India

Email: arvind.gulbake@gmail.com

Received: 01 Mar 2019, Revised and Accepted: 26 Apr 2019

\section{ABSTRACT}

Objective: The present investigation focused on the novel approach using artesunate (AS) loaded mannosylated conjugated multi-walled carbon nanotubes (M-MWCNTs) for site-specific delivery to the brain in the treatment of cerebral malaria (CM).

Methods: The raw MWCNTs were purified by selective oxidation method and then exposed to sequential chemical functionalization according to the following steps: carboxylation, acylation, amine modification and finally, D-mannose conjugation. The AS was loaded via the equilibrium dialysis method in the molar ratio 1:3 of various functionalized sonicated MWCNTs. The functionalized MWCNTs were characterized for elemental analysis, FTIR, TEM, zeta potential and percentage drug entrapment efficiency. The in vitro drug release study was performed on AS conjugated purified MWCNTs (AS-P-MWCNT) and AS conjugated M-MWCNTs. Bio-distribution study was performed on albino rat for quantitative measurement of AS in different organs and blood.

Results: The TEM images of M-MWCNTs indicated their open tubular nature and AS-M-MWCNTs suggests the entrapment of AS. The percent drug entrapment of AS-M-MWCNT was found to be $80.29 \pm 3.4 \%$. In vitro AS release from AS-M-MWCNTs was found in a controlled manner at $\mathrm{pH}$ 7.4. The bio-distribution studies clearly indicate the superiority of the AS-M-MWCNTs, as compared to the plain drug towards increasing the accumulation of AS in brain.

Conclusion: The results suggest that AS-M-MWCNTs could be employed as an efficient nano-carrier for antimalarial therapy in cerebral malaria.

Keywords: Carbon Nanotubes, Cerebral malaria, Artesunate

(C) 2019 The Authors. Published by Innovare Academic Sciences Pvt Ltd. This is an open-access article under the CC BY license (http://creativecommons.org/licenses/by/4.0/) DOI: http://dx.doi.org/10.22159/ijap.2019v11si2.32887

\section{INTRODUCTION}

Malaria is a protozoal disease in humans elicited by protozoan parasites transmitted through female species of Anopheles mosquito of genus Plasmodium. Among five types of parasites plasmodium falciparum is the most virulent and cause serious complication of the infection known as cerebral malaria (CM). The principal mechanism leading to $\mathrm{CM}$ is adherence (mediated by many ligands and receptors) and sequestration of parasitized erythrocytes and immune cells to endothelial cells presents in brain capillaries via induction of an inflammatory process and imbalance in the production of neurotoxic and neuroprotective factors which lead to inflammation of the brain and/or death of humans in severe cases $[1,2]$.

For the management of CM several drugs like quinine or artemisinin [3], are currently used routinely with conventional drug delivery system. The main drawback of conventional malaria chemotherapy is non-specific targeting to intracellular parasites, resulting in higher dose requirements, subsequent intolerable toxicity and the development of multiple drug resistance against parasite. Therefore, an urgent requirement for designing novel drug targets or compounds against this parasite is needed for its management. Formulation and evaluation of novel drug delivery systems is not only less expensive than developing new drugs but may also improve the delivery of existing anti-malarial drugs at the desired rate. Artemisinin derivative has many advantages over other antimalarial drugs, among them commonly used semi-synthetic derivative Artesunate (AS, $\mathrm{C}_{19} \mathrm{H}_{28} \mathrm{O}_{8}$ ) is mainly useful for the multidrug-resistant case of falciparum malaria or Cerebral Malaria (CM). Artemisinin is a sesquiterpene lactone isolated from the plant Artemisia annua and known for its ability to denature the number of plasmodium parasites in the bloodstream and deep organs including the brain by the coagulation of protein present in parasite DNA. It acts on the trophozoite stage of the life cycle of the malaria parasite and stops the formation of schizont.
Carbon nanotubes (CNTs) have established much recent interest as new entities as a novel drug delivery system for experimental disease diagnosis and treatment because of their unique properties to provide a hollow core appropriate for storing guest molecules [4, 5]. Important properties of CNTs, making them a famous tool more than other nanocarriers, are greater stability, biocompatibility, nonimmunogenicity, ease of size alteration and high drug-loading potential [6]. Internal and external surfaces of CNTs can be modified on an individual basis as required and a variety of functional groups can be generated on their surface in support of further conjugation with targeting ligands as well as drug molecules. The CNTs can be also degraded within human brain tissue by myeloperoxidase (MPO) and hydrogen peroxide $\left(\mathrm{H}_{2} \mathrm{O}_{2}\right)$ [7].

The present investigation focuses on the novel approach of using multi-walled carbon nanotubes, which are purified and then surface modified by mannose to target the brain cells having the mannose receptors (transmembrane glycoprotein) expressed by macrophages and binds to mannosylated molecule and mediated their endocytosis to deliver the anti-malarial moiety at desired site and treat the disease in an effective manner. Hence, we are reporting for the first time that mannose conjugated multi-walled carbon nanotubes loaded with anti-malarial drug artesunate to target brain cell serve as a good inhibitor of plasmodium falciparum and can act as a controlled release system.

\section{MATERIALS AND METHODS}

\section{Materials}

Multiwalled carbon nanotubes (MWCNTs) (carbon basis $>90 \%$ ) having diameter $x$ length, i.e. 110-170 $\mathrm{nm}$ x 5-9 $\mu \mathrm{m}$, D-mannose and PTFE filters $(0.45 \mu \mathrm{m}$ pore size) were purchased from SigmaAldrich, USA. Artesunate was obtained as a gift sample from Solisto Pharma Sagar, M. P. INDIA. Dialysis membrane (MWCO, 12-14 KDa) was purchased from Himedia Laboratories Pvt. Ltd. (India). All other 
chemicals and reagents were of analytical grade and deionized water has been used for all experiments.

\section{Purification (of raw MWCNTs)}

By minor modifications the MWCNTs were purified by selective oxidation method [8]. Purification techniques basically, divided into two main streams: Structure selective (to separate the CNTs from the impurities) and size selective separations (provide more homogeneous diameter or size distribution).

\section{Oven purification}

The R-MWCNTs were treated at $250 \pm 2{ }^{\circ} \mathrm{C}$ for $1 \mathrm{hr}$ in hot air oven (Joyti Scientific Industries, India) to get rid of the metallic impurities and amorphous carbon because of elevated temperature to obtain P-MWCNTs.

\section{Acid purification}

After the heat treatment, the P-MWNTs $(500 \mathrm{mg})$ were reacted with concentrated hydrochloric acid under magnetic agitation (Remi, Mumbai, India) for $5 \mathrm{hr}$, and filtered with $0.45 \mu \mathrm{m}$ polytetrafluoroethylene (PTFE) filter. It was used for the elimination of catalytic and amorphous impurities from the unpurified RawMWCNTs to get P-MWCNTs $[9,10]$.

\section{Chemical functionalization}

The P-MWCNTs were subjected to sequential chemical functionalization steps according to the following steps: carboxylation, acylation, amine modification, and mannosylation.

\section{Cutting and carboxylation of MWCNTs}

Carboxylation (cutting and oxidation) of P-MWCNTs were performed to generate carboxyl $(-\mathrm{COOH})$ groups onto the surface and sidewall of MWCNTs. After the heat treatment, the purified MWNTs were refluxed with the acid mixture, i.e. sulphuric acid (98\%) and nitric acid (68\%) $\left(\mathrm{H}_{2} \mathrm{SO}_{4}: \mathrm{HNO}_{3}:: 3: 1 \mathrm{v} / \mathrm{v}\right)$ at $60 \pm 2^{\circ} \mathrm{C}$ for 4 to $6 \mathrm{~h}$ followed by washing through deionized water by centrifugation until the $\mathrm{pH}$ became neutral and filtered by $0.45 \mu \mathrm{m}$ PTFE filters (Hangzhou Anow Microfiltration Co. Ltd., China). The acid treatment was performed under two different reaction conditions- at varying temperatures (25 $\left.{ }^{\circ} \mathrm{C}, 60^{\circ} \mathrm{C}, 80^{\circ} \mathrm{C}, 100{ }^{\circ} \mathrm{C}\right)$ and for different time durations $(1,2,4$ and 6 hr) (tables 1 and 2). After that C-MWCNTs were dried at $100{ }^{\circ} \mathrm{C}$ in a vacuum oven (Jyoti Scientific Industries, Gwalior, India) to remove carbon dioxide and water. During this process, the mixed acid not only grafted functional groups onto the surface of the MWNTs but also dissolved metal particulate impurities. C-MWCNTs (solid) were transferred into a sanction tube (Soniweld, Mumbai) containing water and sonicated for $15 \mathrm{~min}$ to reduce the size of MWCNTs. The percentage yield was calculated by weighing the MWCNTs obtained after drying. For the determination of the acidic sites concentration present on C-MWCNTs were added into unreacted $\mathrm{NaOH}$ was titrated with $0.01 \mathrm{~N}$ hydrochloric acid $(\mathrm{HCl})$ to each sample of carboxylated MWCNTs $[11,12]$.

\section{Acylation of carboxylated MWCNTs}

At $50{ }^{\circ} \mathrm{C}, 300 \mathrm{mg}$ of C-MWCNTs were stirred in $63 \mathrm{ml}$ mixture of thionyl chloride and dimethylformamide (SOCl 2 : DMF: : $60: 3$ ) for $3 \mathrm{~d}$ to get ($\mathrm{CoCl})$ group on the tubes, followed by washing with anhydrous tetrahydrofuran (THF) by centrifuged at $8000 \mathrm{rpm}$ for $20 \mathrm{~min}$ and about 5-6 times for the elimination of excess thionyl chloride. The black remainder of Acy-MWCNTs was fully dried and characterized $[8,13]$.

\section{Amine modification of Acy-MWCNTs}

Half $(150 \mathrm{mg}$ ) of Acy-MWCNTs were reacted with the $70 \mathrm{ml}$ of ethylenediamine (EDA) solution for about $4 \mathrm{~d}$ at $100^{\circ} \mathrm{C}$ to generate$\mathrm{NH}_{2}$ group onto the wall of MWCNTs, followed by cooling at room temperature then washed 5-8 times by ethanol to get rid of the excess diamine from the Am-MWCNTs by repetitive centrifugation. Remainder was dried overnight and characterized [13].

\section{Preparation of mannosylated multiwalled carbon nanotubes}

D-mannose (200 mg) was dissolved in $100 \mathrm{ml}$ sodium acetate buffer ( $\mathrm{pH}$ 4.0 ), followed by heating the mixture at $60 \pm 0.5{ }^{\circ} \mathrm{C}$ for an hour and continuously stirred in a magnetic stirrer (Remi, Mumbai, India) at ambient temperature for $72 \mathrm{~h}$ to ensure the completion of ring opening reaction. From this, $30 \mathrm{mg}$ mannose was added to $10 \mathrm{mg}$ of AmMWCNTs and the mixture was stirred for $3 \mathrm{~d}$ to get the M-MWCNT's. The schematics presentation of mannosylation process is shown in fig. 1 . Purification of M-MWCNTs was done by a dialysis membrane (MWCO $12-14 \mathrm{KDa}$ ) against deionized water for $12 \mathrm{~h}$ to remove detached mannose and other impurities. The volume remained in the dialysis bag centrifuged for $25 \mathrm{~min}$ and dried overnight in a vacuum oven. The characterization of M-MWCNTs was done by FTIR spectroscopy (Perkin-Elmer, USA). Quantitative ninhydrin Kaiser Test was used to determine the degree of mannosylation in M-MWCNTs. The aqueous dispersion of MWCNTs was mixed with two drops of Kaiser A $0.5 \mathrm{ml}$ of $0.065 \% \mathrm{w} / \mathrm{v}$ aqueous KCN solution, with $24.5 \mathrm{ml}$ of dry pyridine and 2.5 $\mathrm{ml}$ of $400 \% \mathrm{w} / \mathrm{v}$ phenol/ethanol mixture) and Kaiser B (5\%, w/v, ethanolic ninhydrin solution) in a microcentrifuge after that heated on a preheated block for 15-30s. The resultant was filtered (Milipore, 0.45 $\mathrm{mm}$ ) and estimated spectrophotometrically (UV-1601, Shimadzu, Japan) $[10,14]$.

\section{Characterization of functionalized MWCNTs}

\section{Elemental analysis}

Elemental analysis of various functionalized MWCNTs were conducted to determine the $\mathrm{S}$ percentage composition in a Flash EA 112 series (Thermo Electron Corp., Italy) (table 3).

\section{Fourier transform infra-red (FTIR) spectroscopy}

FTIR spectroscopy was performed for each step during functionalization to confirm the presence of functional group on MWCNTs. FTIR spectrum were recorded over a scan range of 400$4000 \mathrm{~cm}-1$ (Perkin-Elmer, USA) (fig. 2).

\section{Morphology of MWCNTs and functionalized MWCNTs \\ Transmission electron microscopy}

The size and shape of pristine and functionalized MWCNTs samples were characterized by Transmission Electron Microscopy (TEM) after drying on a copper grid and staining negatively by $1 \%$ phosphotungstic acid (PTA) by the metal shadowing technique [Morgani 268-D, Holland; fig. 3(a-d)].

\section{Zeta potential}

The zeta potential of purified and various functionalized MWCNTs were determined by Malvern Zeta sizer (Malvern Instrument, UK). The zeta potential of raw, purified and various functionalized MWCNTs was determined with $0.05 \mathrm{mg} / \mathrm{ml}$ concentration of MWCNTs suspended in double deionized water ( $\mathrm{pH}$ 7.0) (table 4).

Drug entrapment efficiency of Artesunate in functionalized MWCNTs

The equilibrium dialysis method was used for the loading of drug AS into the P-MWCNTs. Known molar ratio 1:3 of different functionalized sonicated MWCNTs and AS were taken with PBS $(\mathrm{pH}$ 7.4) and incubated for $24 \mathrm{~h}$ with continuous magnetic stirring at $50 \mathrm{rpm}$ (Remi, Mumbai, India). The total mixture was then dialyzed using a dialysis bag (MWCO 12-14 KDa) against 50\% ethanol in PBS ( $\mathrm{pH}$ 7.4) under the sink condition for 30 min to remove unentrapped AS. Amount of entrapped drug was determined indirectly by estimating the amount of free AS at $209 \mathrm{~nm}$ using UV-visible spectrophotometer (Shimadzu-1800, Japan). The formulation of dialysis bag was filtered, lyophilized (Hetro Dry Winner, Germany) and used for further characterization. The same method was followed for the loading of AS in functionalized-MWCNTs. The percentage entrapment of drug in different formulations was calculated using an equation: [15].

$\%$ Entrapment Efficiency

$$
=\frac{(\text { Weight of entraped AS) }}{(\text { Weight of entraped AS }+ \text { Free AS) }} \times 100
$$

\section{In vitro release studies}

The in vitro release of AS from the drug-loaded P-MWCNTs (AS-PMWCNTs) and AS loaded mannosylated MWCNTs (AS-M-MWCNTs) 
was evaluated in PBS, pH 7.4 by equilibrium dialysis tube diffusion technique (MWCO 12-14 KDa; Himedia, India). Briefly, the drug loaded replace by P-MWCNTs and M-MWCNTs. MWCNTs equivalent to $5 \mathrm{mg}$ of the AS was taken in a dialysis tube and dialyzed against $50 \mathrm{ml}$ of release medium at room temperature with constant stirring at $30 \mathrm{rpm}$ on a magnetic stirrer (Remi, India). One ml of the sample was withdrawn at each scheduled time interval and replenished with an equal volume of the fresh medium to maintain the sink condition. The samples were appropriately diluted with the distilled water and were analyzed for AS using UV-visible spectroscopy at $209 \mathrm{~nm}$. Results are shown in (fig. 4).

\section{Tissue bio distribution study}

Albino rats (Wistar strain) were weighed, marked and then divided into 4 groups of 9 each $(n=3)$. Rats were fasted overnight and allowed access to water ad libitum before the experiment. Free AS solution (free AS), AS-P-MWCNTs and AS-M-MWCNTs containing an equivalent dose of AS (1.0 mg/kg body weight) were administered through IV route to rats of first, second and third groups, respectively. The fourth group of animals were kept as control. The animals from each group were sacrificed at 1, 6 and $24 \mathrm{~h}$ time points and the blood were collected via cardiac puncture in anti-clot vials (Himedia, India). Various organs viz. liver, spleen, kidney and brain were carefully excised, isolated, washed with normal saline, were blot dried and stored at $-80^{\circ} \mathrm{C}$ until assay. The tissue biodistribution levels of AS were monitored by UV spectroscopy method. The results were expressed as percentage drug distribution. The data of biodistribution from AS, AS-P-MWCNTs and AS-M-MWCNTs formulations are shown in (fig. 5). All investigations were performed as per the protocol approved by the Institutional Animals Ethical Committee of Adina Institute of Pharmaceutical Sciences, Sagar (M. P.) India.

\section{Statistical analysis}

Statistical test was performed with Graph Pad Instat Software (Version 3.00, Graph Pad Software) by one-way ANOVA followed by
Tukey-Kramer test for multiple comparisons. A probability $p<0.05$ was considered statistically significant.

\section{RESULTS AND DISCUSSION}

Preparation and characterization of mannosylated multiwalled carbon nanotubes

Generally, R-MWCNTs hold several impurities like metals and amorphous carbon in particulate form therefore, strong acid treatment and hot air oven are the two most commonly used techniques for the purification. This removes the amorphous carbon as well as metallic impurities. These impurities were detached to get PMWCNT's by oxidative strong-acid treatment, metals were removed by forming metal oxides and confirmed by elemental analysis.

The strong acid treatment also enhances the nanotubes solubility in aqueous media, separates impurities and shortened MWCNTs [13, 16]. In performing acid treatment of the MWCNTs it was observed that with increasing temperature and time, the length of MWCNTs decreases, hence the time and temperature where optimum length received were found to be $4 \mathrm{~h}$ and $80^{\circ} \mathrm{C}$, respectively (table 1 and 2). The purified MWCNTs were modified to generate more functional groups and then carboxylated by treating with an acidic mixture of sulfuric acid $(98 \%)$ and nitric acid $(68 \%)$ to generate carboxyl ($\mathrm{COOH})$ groups onto the surface and sidewall of nanotubes. The quantification of the number of acidic groups and their corresponding length was determined by acid-base titration and TEM. The C-MWCNT'S were then acylated using thionyl chloride and dimethyl-formamide (DMF) and washed with the anhydrous tetrahydrofuran (THF) for the removal of excess thionyl chloride, for about 5-6 times to produce $(-\mathrm{CoCl})$ groups, and processed to get amine modified MWCNTs by using ethylenediamine; EDA to generate $\left(-\mathrm{NH}_{2}\right)$ group on the surface of the tubes and were washed with ethanol 5-8 times to remove the excess diamine solution by well-known reported methods [12]. The number of free amino groups as determined by quantitative Ninhydrin Kaiser test was found to be $3.1 \mathrm{mmol} / \mathrm{g}$ of amine-modified MWCNTs.

Table 1: Temperature variation for carboxylation of MWCNTs at $1 \mathrm{~h}$

\begin{tabular}{lll}
\hline Temperature $\left({ }^{\circ} \mathbf{C}\right)$ & -COOH concentration $\mathbf{~ m m o l} / \mathbf{g}$ & Length (nm) \\
\hline 25 & $1.4 \pm 0.24$ & $920 \pm 28$ \\
60 & $2.1 \pm 0.36$ & $815 \pm 22$ \\
$\mathbf{8 0}$ & $\mathbf{3 . 5 \pm 0 . 4 5}$ & $\mathbf{7 2 0 \pm 2 4}$ \\
100 & $3.6 \pm 0.53$ & $721 \pm 26$ \\
\hline
\end{tabular}

For mixture of $\mathrm{H}_{2} \mathrm{SO}_{4} / \mathrm{HNO}_{3}$ (3:1). Values represent mean $\pm \mathrm{SD}(\mathrm{n}=3)$.

Table 2: Time variation for carboxylation of MWCNTs at fixed temperature $\left(80^{\circ} \mathrm{C}\right)$

\begin{tabular}{lll}
\hline Time (h) & -COOH concentration mmol/g & Length (nm) \\
\hline 1 & $3.5 \pm 0.45$ & $720 \pm 26$ \\
2 & $7.1 \pm 0.68$ & $450 \pm 10$ \\
4 & $8.5 \pm 0.59$ & $210 \pm 21$ \\
6 & $8.6 \pm 0.62$ & $215 \pm 22$ \\
\hline
\end{tabular}

For mixture of $\mathrm{H}_{2} \mathrm{SO}_{4} / \mathrm{HNO}_{3}(3: 1)$. Values represent mean $\pm \mathrm{SD}(\mathrm{n}=3)$.

Then, mannosylation was carried out by ring opening of mannose followed by reaction of its aldehyde groups with the free AmMWCNTs in $0.1 \mathrm{M}$ sodium acetate buffer ( $\mathrm{pH}$ 4.0) [17]. This leads to the formation of Schiff's base (-N=CH-), which may then get reduced to secondary amines (-NH- $\left.\mathrm{CH}_{2}-\right)$ but stay in equilibrium with Schiff's base. Free mannose and impurities were isolated by dialysis and characterized for the presence of a mannose residue by ligand agglutination assay, i.e. Concanavalin A assay. The mannose conjugation to MWCNTS is schematically shown in fig. 1.

Various treated MWCNTs were heated at $55^{\circ} \mathrm{C}$ to remove carbon dioxide and water. MWCNTs were transferred into a sonication tube (Soniweld, Mumbai) containing water and sonicated for $15 \mathrm{~min}$ (for size reduction). The different functionalized MWCNTs were then subjected to characterization to revel various parameters.
Mannosylation was carried out by coupling amine group present on the surface of MWCNTs. Broad, intense O-H stretch and C-O stretch of mannose around $3400.22 \mathrm{~cm}^{-1}$ and $1641.85 \mathrm{~cm}^{-1}$ respectively, and $\mathrm{N}-\mathrm{H}$ deformation of secondary amine at $1565.87 \mathrm{~cm}^{-1}$ confirmed the Schiff's base formation and some amine formation in the linkage between an aldehyde of mannose and amine termination groups of multi-walled nanotubes.

Elemental analysis of raw MWCNTs, purified, carboxylated, acylated, amine modified and Mannosylated MWCNTs was performed to determine content purity. The carbon content was found to be 97.36\% and $99.12 \%$ for R-MWCNTs and P-MWCNTs respectively, which clearly indicates that no other element was present in PMWCNTs that would affect the integrity and properties of MWCNTs. The increased hydrogen percentage in the case of C-MWCNTs clearly 
suggests that some carboxyl groups have been attached during carboxylation process. The increased nitrogen percentage in the case of Am-MWCNTs clearly suggests that some amine groups have been attached during amine modification of MWCNTs. M-MWCNTs depict increased percentage of hydrogen clearly suggesting attachment of mannose on the carbon surface. Elemental analysis of the MWCNTs exhibiting the percentage of carbon, hydrogen and nitrogen shown in the table 3.

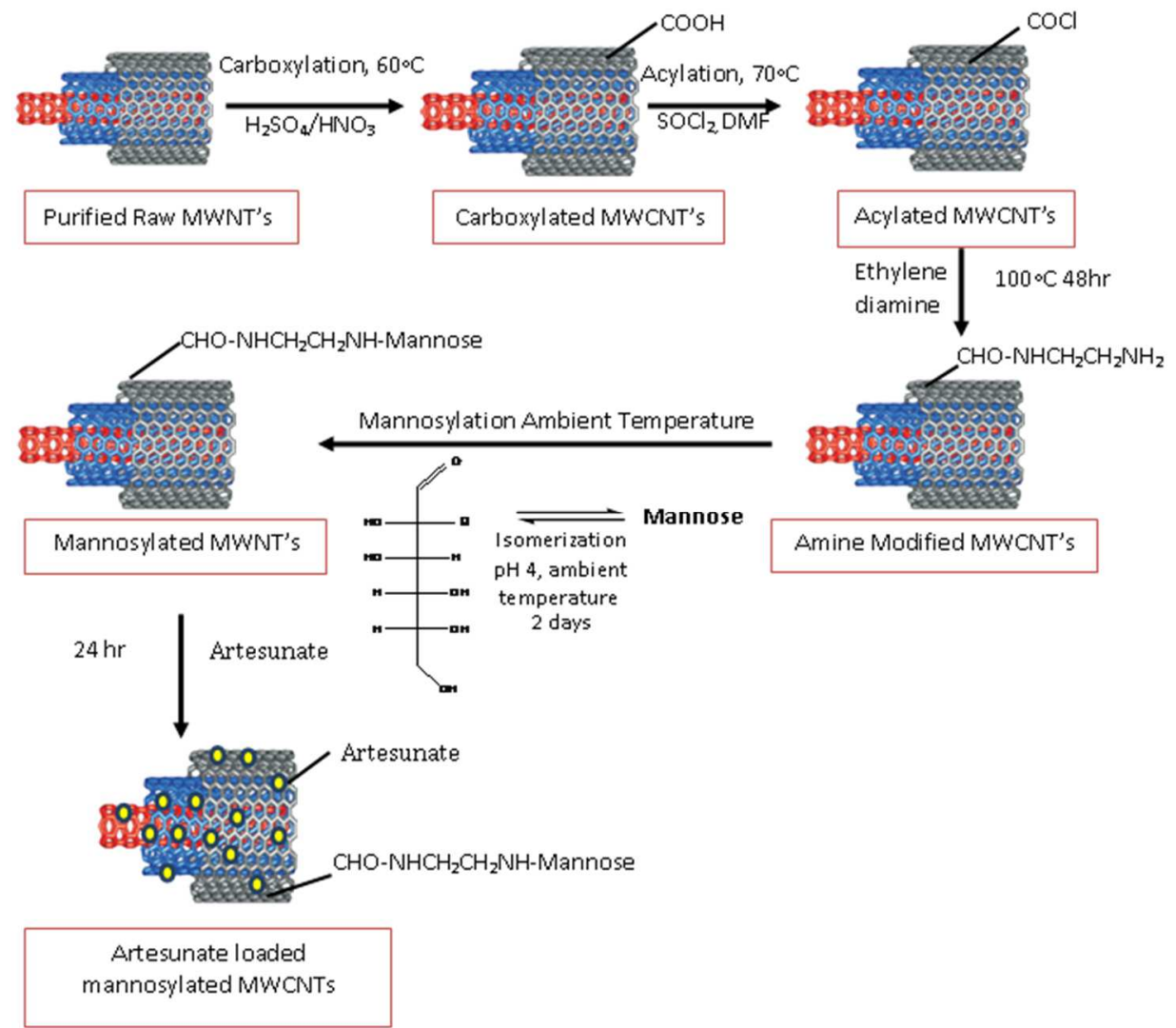

Fig. 1: Schematic representation of MWCNTs functionalization

Table 3: Elemental analysis of different functionalized MWCNTs

\begin{tabular}{llll}
\hline \multirow{2}{*}{ Sample } & \multicolumn{2}{l}{ Atomic percentage \pm SD (\%) } & \\
\cline { 2 - 4 } & Carbon & Hydrogen & Nitrogen \\
\hline Raw-MWCNTs & $92.36 \pm 3.2$ & - & - \\
P-MWCNTs & $99.12 \pm 3.7$ & $1.3 \pm 1.2$ & 1.93 \\
C-MWCNTs & $74.91 \pm 2.6$ & $1.2 \pm 1.1$ & 1.90 \\
Acy-MWCNTs & $70.45 \pm 3.1$ & $3.5 \pm 2.2$ & $8.2 \pm 1.1$ \\
A-MWCNTs & $68.37 \pm 3.5$ & $6.2 \pm 1.3$ & $5.9 \pm 1.2$ \\
M-MWCNTs & $62.39 \pm 3.1$ & & \\
\hline
\end{tabular}

Values represent mean $\pm S D(n=3)$.

\section{Fourier transform infrared spectroscopy}

FTIR study was performed on functionalized MWCNTs to assess the presence of various functional groups over their surface. The PMWCNTs show less dense peaks at $3436.36 \mathrm{~cm}^{-1}, 2853.41 \mathrm{~cm}^{-1}$ and $1120.18 \mathrm{~cm}^{-1}$, which could be ascribed to the $\mathrm{O}-\mathrm{H}$ stretching, $\mathrm{C}-\mathrm{H}$ stretching and $\mathrm{O}-\mathrm{H}$ in-plane bending, respectively (fig. 2). Data confirms the presence of some oxygenated groups generated after the purification process. C-MWCNTs showed few broad, strong peaks at $3416.80 \mathrm{~cm}^{-1}, 2923.44 \mathrm{~cm}^{-1}, 1075.91 \mathrm{~cm}^{-1}, 1639.01 \mathrm{~cm}^{-1}$, $1417.92 \mathrm{~cm}^{-1}$ and $1025.9 \mathrm{~cm}^{-1}$, which could be ascribed to $\mathrm{O}-\mathrm{H}$ stretching, $\mathrm{C}-\mathrm{H}$ stretches, $\mathrm{C}=\mathrm{O}$ stretching, $\mathrm{C}=\mathrm{C}$ stretching, $\mathrm{C}-\mathrm{O}$ is stretching and $\mathrm{O}-\mathrm{H}$ bending, respectively (fig. 2). The data confirm the presence of carboxylic $(-\mathrm{COOH})$ groups present on the surface of MWCNTs. As functionalization proceeds numbers of peaks increases due to the further attachment of other functional groups. FTIR analysis proved the formation of Schiff's base and secondary amine $\left(-\mathrm{NH}^{-} \mathrm{CH}_{2}-\right)$ linkage between aldehyde group of mannose and Am-
MWCNTs. M-MWCNTs showed the peak at $1567.80 \mathrm{~cm}^{-1}, 1646.66$ $\mathrm{cm}^{-1}, 2925.64 \mathrm{~cm}^{-1}$, and $3401.53 \mathrm{~cm}^{-1}$, which could be ascribed to$\mathrm{C}=\mathrm{N}$ stretching (Schiff 's base formation), $\mathrm{C}-\mathrm{H}$ stretch of $\mathrm{CH}_{2}, \mathrm{O}-\mathrm{H}$ stretching respectively (fig. 2). AS-M-MWCNTs showed the peak at $1587.80 \mathrm{~cm}^{-1}, 1646.66 \mathrm{~cm}^{-1}, 1379.45 \mathrm{~cm}^{-1}$ and $2949.18 \mathrm{~cm}^{-1}$ which could be ascribed to $-\mathrm{C}=\mathrm{N}$ stretching due to Shiff's base, $-\mathrm{C}=\mathrm{O}$ stretch of amide bond, $\mathrm{C}=\mathrm{O}$ bond, $\mathrm{C}-\mathrm{H}$ stretch of $\mathrm{CH}_{2}$ respectively (fig. 2).

\section{Zeta potential}

The R-MWCNTs shows positive zeta potential whereas P-MWCNTs shows a negative value of zeta potential $(-44.5 \mathrm{mV})$, which could be due to the presence of carboxylic group during the purification process. AcyMWCNTs showed negative value due to the negative inductive effect of chlorine. Similarly, the Am-MWCNTs shows a positive value due to protonation of the amino group. The M-MWCNTs shows comparatively lower, but still positive value of the zeta potential. The change in zeta potential strictly depends upon the $\mathrm{pH}$ of the medium (table 4). 
Table 4: Zeta potential and drug entrapment efficiency of MWCNTs

\begin{tabular}{lll}
\hline Types of MWCNTs & Zeta potential $(\mathbf{m V})$ & Percent entrapment efficiency of AS \\
\hline Raw MWCNTs & $+41.6 \pm 1.14$ & NA \\
P-MWCNTs & $-44.5 \pm 1.86$ & $54.35 \pm 2.23$ \\
C-MWCNT & $-41.7 \pm 2.12$ & $68.45 \pm 1.25$ \\
Acy-MWCNT & $-17.2 \pm 0.76$ & $62.22 \pm 1.36$ \\
Am-MWCNTs & $+24.9 \pm 0.87$ & $55.03 \pm 1.41$ \\
M-MWCNTs & $+1.730 \pm 0.02$ & $80.29 \pm 1.40$ \\
\hline
\end{tabular}

Values represent mean $\pm \mathrm{SD}(\mathrm{n}=3)$.
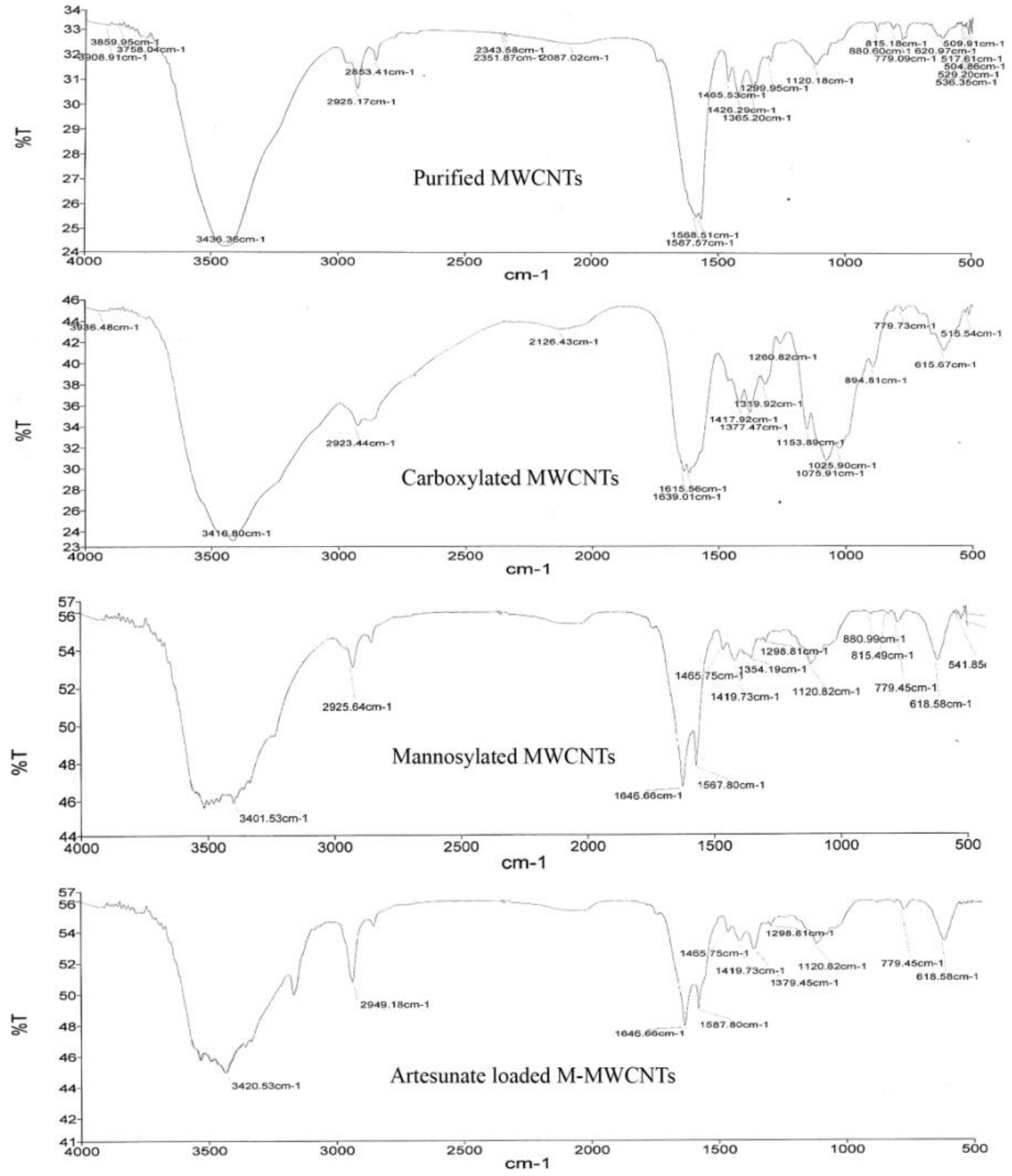

Fig. 2: FTIR spectrums of functionalized MWCNTs

\section{Transmission electron microscopy}

The lengths of R-MWCNTs, C-MWCNTs, M-MWCNTs and AS-M-MWCNTs were investigated by transmission electron microscope (TEM). CMWCNTs showed a reduction of length of MWCNTs due to oxidation and formation of $-\mathrm{COOH}$ groups. During strong-acid treatment ends of
MWCNTs, five-membered carbon rings and other defect sites result in the generation of - $\mathrm{COOH}$ group and cutting of MWCNTs. Hence, the TEM photographs showed the length of carboxylated and mannosylated MWCNTs to be around $500 \mathrm{~nm}$ as depicted in fig. 3 (b) and (c). The images of M-MWCNTs indicated their open tubular nature and AS-MMWCNTs suggests the entrapment of AS (fig. 3). 

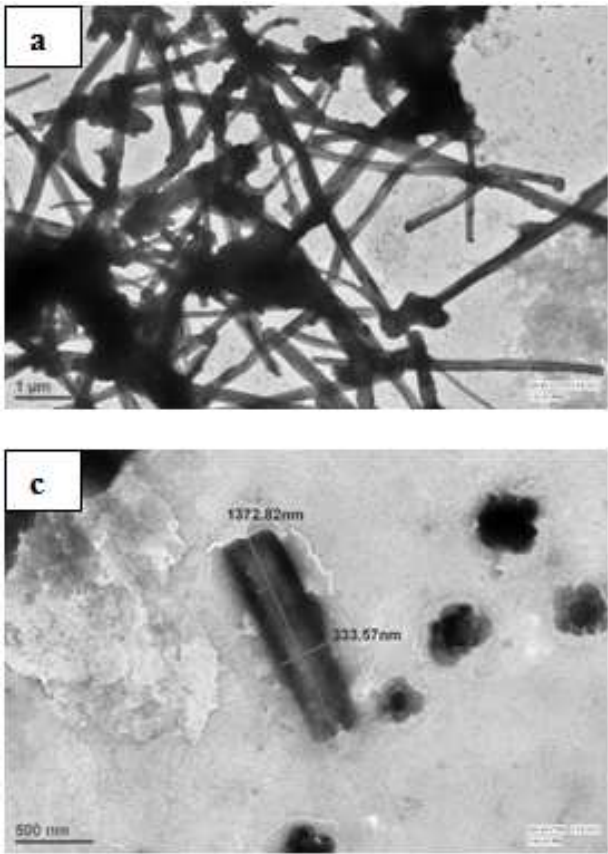
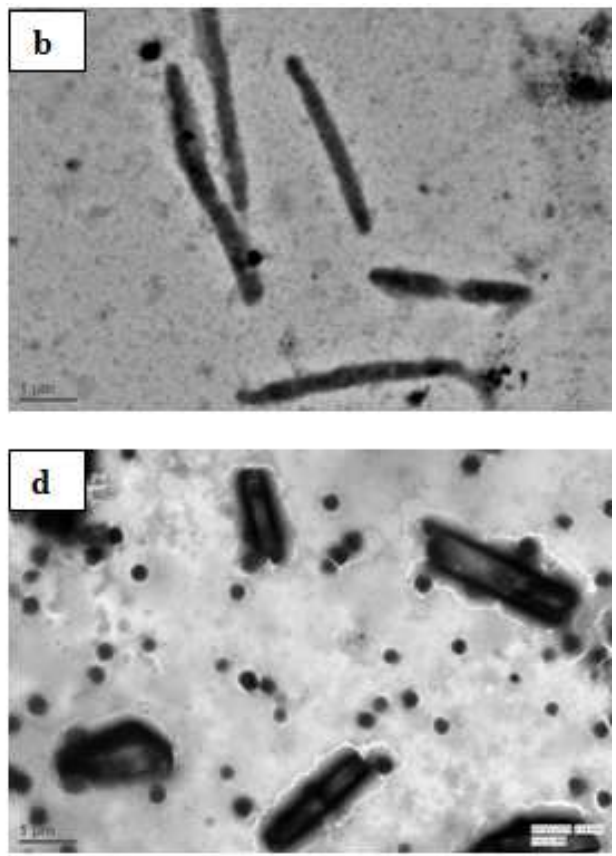

Fig. 3: TEM images of MWCNTs at (a) R-MWCNTs (b), C-MWCNTs and (c) M-MWCNTs (d) AS-M-MWCNTs

\section{Percent drug entrapment efficiency}

It was already reported that the drug can be easily adsorbed on sidewalls and surface of the CNTs ( $\pi-\pi$ stacking interactions) by mixing the drug with CNTs. Similarly AS molecules were entrapped in MWCNTs through $\pi-\pi$ stacking as well as electrostatic interactions, which seem to be more stable in PBS [18]. Entrapment efficiency of AS in purified, carboxylated, acylated, amine modified and mannosylated MWCNTs was found to be $54.35 \pm 2.23$, $68.45 \pm 1.25,62.22 \pm 1.36,55.03 \pm 1.41$ and $80.29 \pm 1.40$ respectively (table 4). The P-MWCNTs were present in the bundle state or in aggregates and very little surface area was available for the entrapment of drug in bundled MWCNTs which shows limited entrapment for AS-P-MWCNTs. However, C-MWCNTs showed a large amount of entrapped drug due to the opening of bundles of purified raw MWCNTs and presence of various pores generated by acid treatment which provides larger surface area and easy penetration into inner cavity of C-MWCNTs. While comparing the percent drug entrapment within different f-MWCNTs, the MMWCNTs showed the highest percentage of AS entrapment and excellent dispersibility due to debundling.

\section{In vitro release}

The in vitro release of AS from AS-P-MWCNTs and AS-M-MWCNTs was monitored under physiological conditions PBS (pH 7.4). The initial burst release achieved due to diffusion or the adsorbed AS on the surface of MWCNTs. It was observed that the release of AS was found to be sustained at PBS pH 7.4 and about $96.46 \%$ of AS was released in $100 \mathrm{~h}$, possibly due to the presence of bulky mannose chain around the MWCNTs, which poses a hindrance to the drug release, compared to P-MWCNTs (fig. 4).

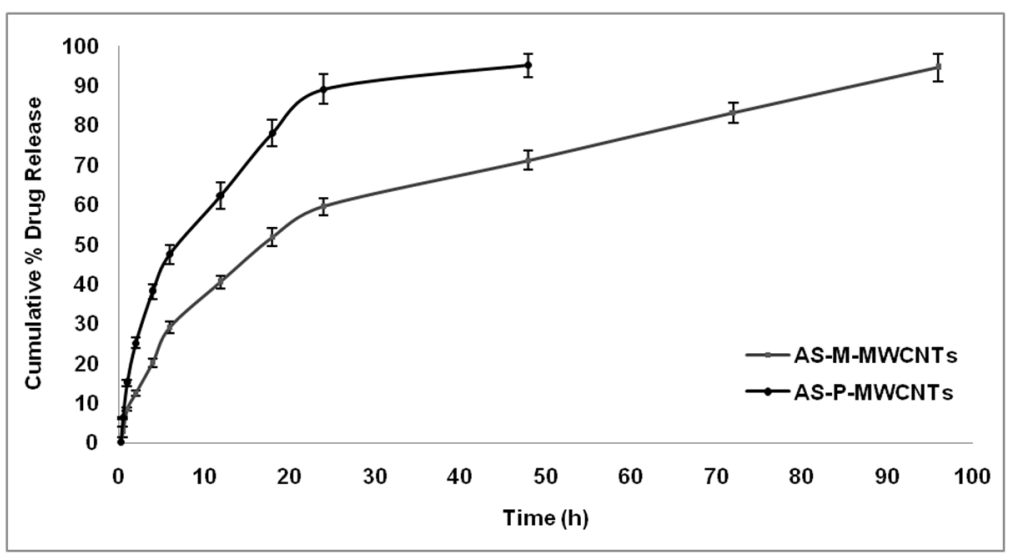

Fig. 4: Cumulative percentage drug release from AS-P-MWCNTs and AS-M-MWCNT's in PBS $(\mathrm{pH} 7.4)$ at $37^{\circ} \mathrm{C}(\mathrm{n}=3)$. Each bar represents mean $\pm \operatorname{SD}(n=3)$

\section{Tissue biodistribution study}

A biodistribution study was performed for free AS solution, AS-PMWCNTs and AS-M-MWCNTs formulations administered through IV route to compare the amount of AS that accumulates in various tissue like liver, brain, kidney and spleen at different time intervals. Amount of AS present in blood at various time intervals was also determined. One hr post IV administration of free AS solution $(1 \mathrm{mg} / \mathrm{kg})$, the 
amount of drug present in blood was found to be very high along with accumulation in liver and spleen. AS was found to be rapidly cleared from blood within $24 \mathrm{~h}$ and the AS level in liver and spleen declined over the time and very low accumulation observed in the brain. In case of AS-M-MWCNTs increased level of AS $(16.3 \pm 0.46 \%)$ accumulation observed in brain after $6 \mathrm{~h}$ and found even higher $(22.4 \pm 0.78 \%)$ at 24 $\mathrm{h}$, which may be due to receptor-mediated endocytosis. It was inferred that AS-M-MWCNTs were highly accumulated in brain and provided a sustained release of drug as compare to free AS and AS-P-MWCNTs. The biodistribution studies (fig. 5) clearly indicate the superiority of the AS-M-MWCNTs, as compared to the plain drug towards increasing the accumulation of AS in brain.

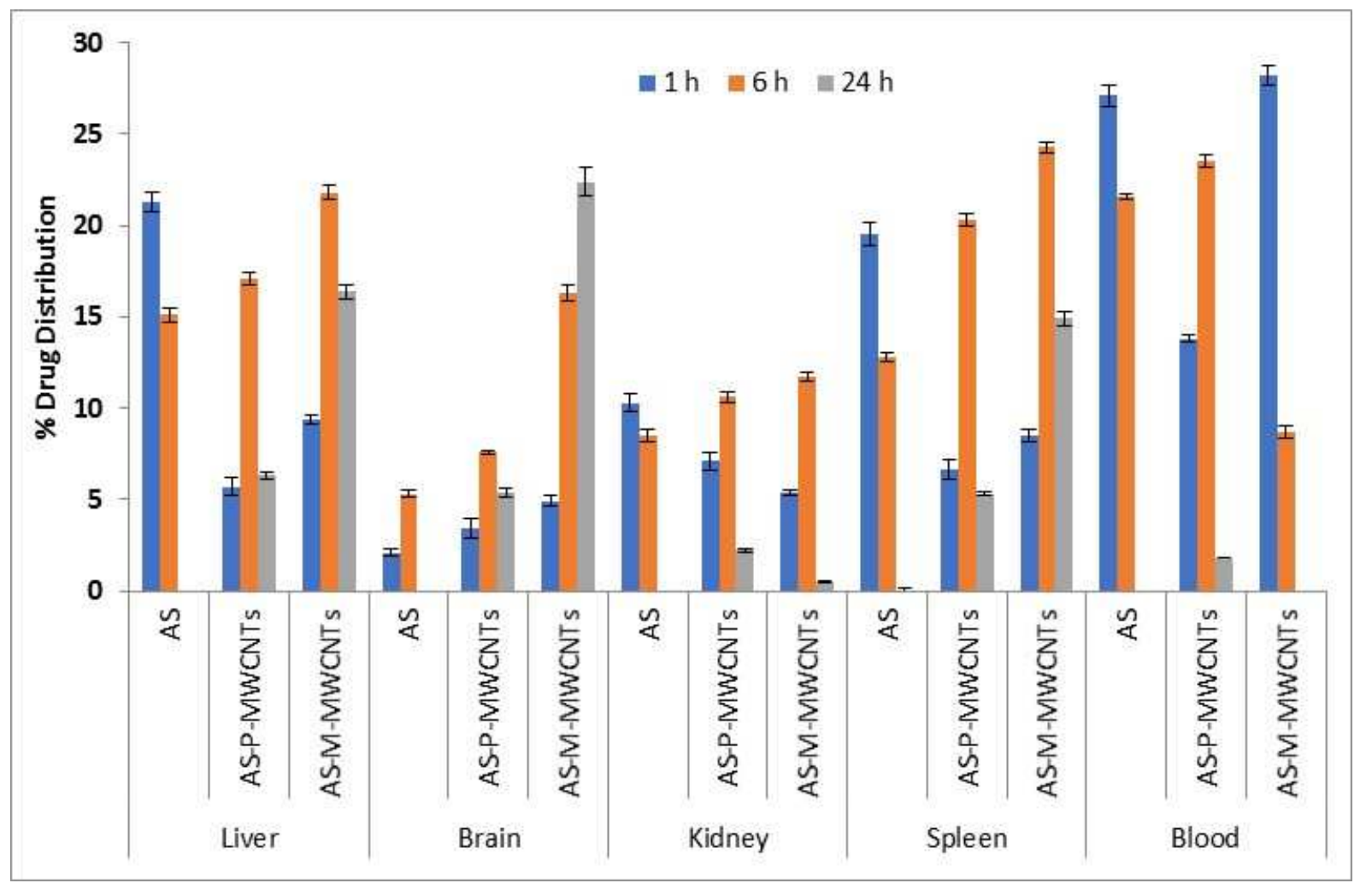

Fig. 5: Tissue biodistribution study of AS at $1 \mathrm{~h}, 6 \mathrm{~h} \& 24 \mathrm{~h}$ after i.v. injection of free AS, AS-P-MWCNTs and AS-M-MWCNTs in Albino rat.

\section{CONCLUSION}

The main problem in the treatment of CM is the ineffective amount of drug could reach to the brain due to lower shelf life in blood. In the present investigation AS loaded M-MWCNTs were developed to target the brain cells using the mannose receptor present at the membrane of various brain cells. From this study, it can be concluded that the AS loaded M-MWCNTs formulation showed efficient AS release to the desired site with an improved therapeutic margin of safety and an effective and alternative nano-formulation for the treatment of Cerebral Malaria in near future.

\section{ACKNOWLEDGMENT}

Authors are thankful to the Science and Engineering Research Board (SERB), New Delhi, India, for financial support through research project file No. EEQ/2016/000789.

\section{ABBREVIATION}

MWCNTs $=$ Multiwalled carbon nanotubes, R-MWCNTs $=$ Raw MWCNTs, P-MWCNTs = Purified-MWCNTs, C-MWCNTs = Carboxylated MWCNTs, Acy-MWCNTs = Acylated MWCNTs, AmMWCNTs $=$ Amine Modified MWCNTs, M-MWCNTs $=$ Mannosylated MWCNTs, AS-MWCNTs = Artesunate loaded MWCNTs, R-6G MWCNTs = Rhodamine-6G loaded MWCNTs, myeloperoxidase (MPO)

\section{AUTHORS CONTRIBUTIONS}

First, second and corresponding authors contributed equally. Third and fourth authors helped in data analysis and manuscript writing.

\section{CONFLICTS OF INTERESTS}

\section{Declared none}

\section{REFERENCES}

1. Simner P. Medical parasitology taxonomy update. I Clin Microbiol 2017;55:43-7.

2. Golenser J, McQuillan J, Hee L, Mitchell AJ, Hunt NH. Conventional and experimental treatment of cerebral malaria. Int J Parasitol 2006;36:583-93.

3. Gay F, Zougbede S, N'Dilimabaka N, Rebollo A, Mazier D, Moreno A. Cerebral malaria: what is known and what is on research. Rev Neurol (Paris) 2012;168:239-56.

4. Iijima S. Helical microtubules of graphitic carbon. Nature 1991. p. 56-8.

5. Davis JJ, Coleman KS, Azamian BR, Bagshaw CB, Green ML. Chemical and biochemical sensing with modified single walled carbon nanotubes. Chem Eur J 2003;9:3732-9.

6. Martin CR, Kohli P. The emerging field of nanotube biotechnology. Nat Rev Drug Discovery 2003;2:29-37.

7. Sajid MI, Jamshaid U, Jamshaid T, Zafar N, Fessi H, Elaissari A. Carbon nanotubes from synthesis to in vivo biomedical applications. Int J Pharm 2016;501:278-99.

8. Shen J, Huang W, Wu L, Hu Y, Ye M. Thermo-physical properties of epoxy nanocomposites reinforced with amino-functionalized multi-walled carbon nanotubes. Composites Part A: Appl Sci Manufacturing 2007;38:1331-6.

9. Lodhi N, Mehra NK, Jain NK. Development and characterization of dexamethasone mesylate anchored on multi-walled carbon nanotubes. J Drug Target 2013;21:67-76. 
10. Pruthi J, Mehra NK, Jain NK. Macrophages targeting of amphotericin B through mannosylated multiwalled carbon nanotubes. J Drug Target 2012;20:593-604.

11. Gong B, Ikematsu A, Waki K. Impacts of structure defects and carboxyl and carbonyl functional groups on the work function of multiwalled carbon nanotubes. Carbon 2017;114:526-32.

12. Jain AK, Dubey V, Mehra NK, Lodhi N, Nahar M, Mishra DK, et al. Carbohydrate-conjugated multiwalled carbon nanotubes: development and characterization. Nanomedicine 2009;5:432-42.

13. Jain AK, Kumar Mehra N, Lodhi N, Dubey V, Mishra DK, Jain PK, et al. Carbon nanotubes and their toxicity. Nanotoxicology 2007;1:167-97.

14. Nahar M, Jain NK. Preparation, characterization and evaluation of targeting potential of amphotericin B-loaded engineered PLGA nanoparticles. Pharm Res 2009;26:2588-98.
15. Ma PC, Kim JK, Tang BZ. Functionalization of carbon nanotubes using a silane coupling agent. Carbon 2006;44:3232-8.

16. Wu W, Wieckowski S, Pastorin G, Benincasa M, Klumpp C, Briand JP, et al. Targeted delivery of amphotericin B to cells by using functionalized carbon nanotubes. Angewandte Chemie International Edition 2005;44:6358-62.

17. Jain A, Agarwal A, Majumder S, Lariya N, Khaya A, Agrawal H, et al. Mannosylated solid lipid nanoparticles as vectors for sitespecific delivery of an anti-cancer drug. J Controlled Release 2010;148:359-67.

18. Liu Z, Winters M, Holodniy M, Dai H. siRNA delivery into human $t$ cells and primary cells with carbon-nanotube transporters. Angewandte Chemie International Edition 2007;46:2023-7. 